\title{
Partitions into large unequal parts
}

\author{
Kevin Fergusson
}

Let $u=\left(u_{j}\right)_{1}^{\infty}$ be a strictly increasing sequence of positive integers and for $x \geqslant 1$ let $U(x)$ be the number of terms of $u$ which do not exceed $x$. For integers $m$ and $n$ such that $0 \leqslant m<n / 2$ define $q_{u}(m, n)$ to be the number of partitions of $n$ into distinct parts coming from the sequence $u$ and exceeding $m$.

In the special case when $u$ is the sequence of positive integers, the classical function $q(n)=q_{u}(0, n)$ and, more recently, the function $q(m, n)=q_{u}(m, n)$ have been investigated by several authors. Freiman and Pitman [1] have recently given asymptotic estimates for $q(m, n)$ as $n \rightarrow \infty$.

In the general case the function $q_{u}(m, n)$ has also been studied, mainly for $m=0$. In particular, Roth and Szekeres [2] have given an asymptotic formula for $q_{u}(0, n)$ which is widely applicable.

This thesis studies the asymptotic behaviour of $q_{u}(m, n)$ as $n \rightarrow \infty$ for sequences such that $U(x) \sim C_{0} x^{s}(\log x)^{-t}$ as $x \rightarrow \infty$, where $C_{0}>0, s>0$ and $t \geqslant 0$ are constants. Chapter 1 introduces the problem and provides historical background and Chapter 2 gives auxiliary results.

Chapter 3 presents the main theorem. For $u$ as above satisfying a suitable further condition, and for given small positive $\delta$, this gives an asymptotic estimate for $q_{u}(m, n)$ which is valid uniformly in $m$ such that $0 \leqslant m \leqslant n^{1-\delta}$ as $n \rightarrow \infty$. The result is motivated by probabilistic considerations similar to those of [1] and the proof uses the circle method as in $[\mathbf{1}]$.

The next two chapters cover applications of the main theurem. The first part of Chapter 4 shows that the theorem applies to three wide classes of sequences which together include all the specific examples in [2]. The remainder of the chapter shows that under the conditions of the main theorem, for relatively small $m$, we have, as $n \rightarrow \infty$

$$
q_{u}(m, n) \sim 2^{-U(m)} q_{u}(0, n)
$$

Received 11th March, 1997

Thesis submitted to the Adelaide University, March 1996. Degree approved, October 1996. Supervisor: Dr Jane Pitman.

Copyright Clearance Centre, Inc. Serial-fee code: 0004-9729/97 $\$ A 2.00+0.00$. 
Chapter 5 uses the main theorem to obtain precise results about $q_{u}(m, n)$ in the case when $u$ is the sequence of $k$-th powers.

Chapters 6 and 7 are devoted to more detailed study of the case when $u$ is the sequence of positive integers. This work extends the results of [1].

\section{REFERENCES}

[1] G.A. Freiman and J. Pitman, 'Partitions into distinct large parts', J. Austral. Math. Soc. Ser. A 57 (1994), 386-416.

[2] K.F. Roth and G. Szekeres, 'Some asymptotic formulae in the theory of partitions', Quart. J. Math. Oxford Ser. (2) 5 (1954), 241-259.

PO Box N435

Grosvenor Place, NSW 1220

Australia 